\title{
Noncummutative Decrumpling Inflation and Running of the Spectral Index
}

\author{
Forough Nasseri \\ Department of Physics, Tarbiat Moallem University of Sabzevar, \\ P.O.Box 397, Sabzevar, Iran \\ and \\ Khayyam Planetarium, P.O.Box 844, Neishabour, Iran \\ E-mail: nasseri@fastmail.fm
}

\author{
S. A. Alavi \\ Department of Physics, Tarbiat Moallem University of Sabzevar, \\ P.O.Box 397, Sabzevar, Iran \\ E-mail: alavi@sttu.ac.ir
}

\begin{abstract}
We present a new inflation model, known as noncommutative decrumpling inflation, in which space has noncommutative geometry with time variability of the number of spatial dimensions. Within the framework of noncommutative decrumpling inflation, we compute both the spectral index and its running. Our results show the effects of both time variability of the number of spatial dimensions and noncommutative geometry on the spectral index and its running. Two classes of examples have been studied and comparisons made with the standard slow-roll formulae. We conclude that the effects of noncommutative geometry on the spectral index and its running are much smaller than the effects of time variability of spatial dimensions.
\end{abstract}

KEYWORDS: Inflation; noncommutative geometry; extra dimensions.

Report number: STTU-Phys-02-2004. 


\section{Contents}

$\begin{array}{ll}\text { 1. Introduction } & 1\end{array}$

2. Review of Decrumpling or TVSD Model 3

2.1 Relation between the effective space dimension $D(t)$ and characteristic size of the Universe $a(t)$

2.2 Physical meaning of $D_{P}$

3. Running of the Spectral Index in Noncommutative Decrumpling In$\begin{array}{ll}\text { flation } & 6\end{array}$

3.1 Decrumpling chaotic inflation 6

3.2 Explicit formulae for running in noncommutative decrumpling inflation 7

3.3 The first example: $V(\phi)=\frac{1}{2} m^{2} \phi^{2} \quad 9$

3.4 The second example: $V(\phi)=\lambda \phi^{4} \quad 10$

4. Conclusions 12

\section{Introduction}

In nature there are three spatial dimensions and one time dimension. The idea of having space-time dimensions other than $3+1$ goes back to Kaluza-Klein theory. This concept has been generalized to string theory with space dimension more than three but still an integer and a constant.

We here present a new inflation model, known as noncommutative decrumpling inflation model in which not only the number of spatial dimension has a dynamical behavior and decreases but also space has a noncommutative geometry.

Our motivation to study noncommutative decrumpling inflation is to investigate cosmological implications of both time variability of the number of spatial dimensions and noncommutativity of space. To do so, we compute the spectral index and its running within the framework of noncommutative decrumpling inflation. For more details about a model Universe with time variable space dimensions (TVSD), known as TVSD or decrumpling model, see Refs. [1-7]. 
This article is a follow-up paper of Ref. [4] about TVSD or decrumpling chaotic inflation model. To do this research an important conceptual issue is properly dealt with the meaning of time variability of the number of spatial dimensions. In Ref. [2], this conceptual issue has been discussed in detail.

Although time variability of spatial dimensions have not been firmly achieved in experiments and theories, such dynamical behavior of the spatial dimensions should not be ruled out in the context of cosmology and astroparticle physics.

Here, we will be concerened with the approaches proposed in the pioneer paper[1] where the cosmic expansion of the Universe is named decrumpling expansion and is due to decrease of the number of spatial dimensions. The most important difference between decrumpling model and other attempts about the time evolution of spatial dimension is that in this model the number of extra spatial dimensions changes with time while in other theories the size of extra spatial dimensions is a dynamical parameter [8, 9]. Based on time variability of the size of spatial dimensions it has been reported[9] that the present rate of change of the mean radius of any additional spatial dimensions to be less than about $10^{-19} \mathrm{yr}^{-1}$. It is worth mentioning that the result of Ref. [9] is based on dynamical behavior of the size of extra spatial dimensions while in decrumpling model we take the size of extra spatial dimensions to be constant and the number of spatial dimensions decreases continuously as the Universe expands. The present rate of time variation of the number of the spatial dimensions in decrumpling or TVSD model is about $10^{-13} \mathrm{yr}^{-1}$.

Another subject which lately has attracted much attention is noncommutative spaces. It is generally believed that the picture of space-time as a manifold should break down at very short distances of the order of the Planck length. Field theories on noncommutative spaces may play an important role in unraveling the properties of nature at the Planck scale. It has been shown that the noncommutative geometry naturally appears in string theory with a non zero antisymmetric B-field [10].

Besides the string theory arguments the noncommutative field theories by themselves are very interesting. In a noncommutative space-time the coordinates operators satisfy the commutative relation

$$
\left[\hat{x}^{\mu}, \hat{x}^{\nu}\right]=i \theta^{\mu \nu}
$$

where $\hat{x}$ are the coordinate operators and $\theta^{\mu \nu}$ is an antisymmetric tensor of dimension of (length) ${ }^{2}$. Generally noncommutative version of a field theory is obtained by replacing the product of the fields appearing in the action by the star product

$$
(f \star g)(x)=\left.\exp \left(\frac{i}{2} \theta^{\mu \nu} \frac{\partial}{\partial x^{\mu}} \frac{\partial}{\partial y^{\nu}}\right) f(x) g(y)\right|_{x=y}
$$


where $f$ and $g$ are two arbitrary functions which we assume to be infinitely differentiable.

In recent years there have been a lot of works devoted to the study of noncommutative field theory or noncommutative quantum mechanics and possible experimental consequences of extensions of the standard formalism [11]-[19]. In the last few years there has been also a growing interest in probing the space-space noncommutativity effects on cosmological observations [20]-[25].

We will use the natural units system that sets $k_{B}, c$, and $\hbar$ all equal to one, so that $\ell_{P}=M_{P}^{-1}=\sqrt{G}$. To read easily this article we also use the notation $D_{t}$ instead of $D(t)$ that means the space dimension $D$ is as a function of time.

The plan of this article is as follows. In section 2, we give a brief review of decrumpling model in commutative spaces. In section 3 , we first present the explicit and general formulae for the spectral index and its running within the framework of noncommutative decrumpling inflation and then apply them to two classes of examples of the inflaton potential. Finally, we discuss our results and conclude in section 4 .

\section{Review of Decrumpling or TVSD Model}

Decrumpling model is based on the assumption that the basic blocks of the space-time are fractaly structured $[1,2]$. In the pioneer paper [1] the spatial dimension of the Universe was considered as a continuous time dependent variable. As the Universe expands, its spatial dimension decreases continuously, thereby generating what has been named a decrumpling Universe. Then this model has been overlooked and the quantum cosmological aspects, as well as, a possible test theory for studying time evolution of Newton's constant have also been discussed [2,3]. Chaotic inflation in decrumpling model and its dynamical solutions have also been studied $[4,5]$.

The concept of decrumpling expansion of the Universe is inspired by the idea of decrumpling coming from polymer physics [1,2]. In this model the fundamental building blocks of the Universe are like cells with arbitrary dimensions having in each dimension a characteristic size $\delta$ which maybe of the order of the Planck length $\mathcal{O}\left(10^{-33}\right.$ $\mathrm{cm}$ ) or even smaller so that the minimum physical radius of the Universe is $\delta$. These "space cells" are embedded in a $\mathcal{D}$ space, where $\mathcal{D}$ may be up to infinity. Therefore, the space dimensions of the Universe depend on how these fundamental cells are configured in this embedding space. The Universe may have begun from a very crumpled state having a very high dimension $\mathcal{D}$ and a size $\delta$, then have lost dimension through a uniform decrumpling which we see like a uniform expansion. The expansion of space, being now understood like a decrumpling of cosmic space, reduces the space-time dimension continuously from $\mathcal{D}+1$ to the present value $D_{0}+1$. In this picture, the Universe can 
have any space dimension. As it expands, the number of spatial dimensions decreases continuously. The physical process that causes or necessitates such a decrease in the number of spatial dimensions comes from how these fundamental cells are embedded in a $\mathcal{D}$ space.

As an example, take a limited number of small three-dimensional beads. Depending on how these beads are embedded in space they can configure to a one-dimensional string, two-dimensional sheet, or three-dimensional sphere. This is the picture we are familiar with from the concept of crumpling in polymer physics where a crumpled polymer has a dimension more than one. Or take the picture of a clay which can be like a three-dimensional sphere, or a two-dimensional sheet, or even a one-dimensional string, a picture based on the theory of fluid membranes.

While it is common to make ad hoc assumptions in cosmological model building in the absence of a complete theory of quantum gravity, some of the particular ingredients of the model owe their physical basis perhaps more to polymer physics than to cosmology. Progress with decrumpling model can only be made if there is a breakthrough in terms of finding a natural mechanism for varying the number of spatial dimensions in some alternative fashion to that which is considered here.

For more details about motivation for choosing this model and its technical and conceptual formalism, see Ref. [2].

\subsection{Relation between the effective space dimension $D(t)$ and characteristic size of the Universe $a(t)$}

Assume the Universe consists of a fixed number $\mathbb{N}$ of universal cells having a characteristic length $\delta$ in each of their dimensions. The volume of the Universe at the time $t$ depends on the configuration of the cells. It is easily seen that $[1,2]$

$$
\operatorname{vol}_{D_{t}}(\text { cell })=\operatorname{vol}_{D_{0}}(\operatorname{cell}) \delta^{D_{t}-D_{0}}
$$

where the $t$ subscript in $D_{t}$ means that $D$ to be as a function of time, i.e. $D(t)$. In previous references [1]-[7] the notation of $D_{t}$ did not use for $D(t)$.

Interpreting the radius of the Universe, $a$, as the radius of gyration of a crumpled "universal surface", the volume of space can be written $[1,2]$

$$
\begin{aligned}
a^{D_{t}} & =\mathbb{N}_{\operatorname{vol}_{D_{t}}(\text { cell })} \\
& =\mathbb{N}_{\operatorname{vol}_{D_{0}}(\text { cell }) \delta^{D_{t}-D_{0}}} \\
& =a_{0}{ }^{D_{0}} \delta^{D_{t}-D_{0}}
\end{aligned}
$$

or

$$
\left(\frac{a}{\delta}\right)^{D_{t}}=\left(\frac{a_{0}}{\delta}\right)^{D_{0}}=e^{C}
$$


where $C$ is a universal positive constant. Its value has a strong influence on the dynamics of space-time, for example on the dimension of space, say, at the Planck time. Hence, it has physical and cosmological consequences and may be determined by observation. The zero subscript in any quantity, e.g. in $a_{0}$ and $D_{0}$, denotes its present value. We coin the above relation as a "dimensional constraint" which relates the "scale factor" of decrumpling model to the spatial dimension. We consider the comoving length of the Hubble radius at present time to be equal to one. So the interpretation of the scale factor as a physical length is valid. The dimensional constraint can be written in this form $[1,2]$

$$
\frac{1}{D_{t}}=\frac{1}{C} \ln \left(\frac{a}{a_{0}}\right)+\frac{1}{D_{0}} .
$$

It is seen that by the expansion of the Universe, the space dimension decreases. Time derivative of (2.3) or (2.4) leads to

$$
\dot{D}_{t}=-\frac{D_{t}^{2} \dot{a}}{C a}
$$

It can be easily shown that the case of constant space dimension corresponds to when $C$ tends to infinity. In other words, $C$ depends on the number of fundamental cells. For $C \rightarrow+\infty$, the number of cells tends to infinity and $\delta \rightarrow 0$. In this limit, the dependence between the space dimensions and the radius of the Universe is removed, and consequently we have a constant space dimension $[1,2]$.

\subsection{Physical meaning of $D_{P}$}

We define $D_{P}$ as the space dimension of the Universe when the scale factor is equal to the Planck length $\ell_{P}$. Taking $D_{0}=3$ and the scale of the Universe today to be the present value of the Hubble radius $H_{0}^{-1}$ and the space dimension at the Planck length to be 4,10 , or 25 , from Kaluza-Klein and superstring theory, we can obtain from (2.3) and (2.4) the corresponding value of $C$ and $\delta$

$$
\begin{aligned}
\frac{1}{D_{P}} & =\frac{1}{C} \ln \left(\frac{\ell_{P}}{a_{0}}\right)+\frac{1}{D_{0}}=\frac{1}{C} \ln \left(\frac{\ell_{P}}{H_{0}^{-1}}\right)+\frac{1}{3}, \\
\delta & =a_{0} e^{-C / D_{0}}=H_{0}^{-1} e^{-C / 3} .
\end{aligned}
$$

In Table 1 , values of $C, \delta$ and also $\left.\dot{D}_{t}\right|_{0}$ for some interesting values of $D_{P}$ are given.[2][5]. These values are calculated by assuming $D_{0}=3$ and $H_{0}^{-1}=3000 h_{0}^{-1} \mathrm{Mpc}=$ $9.2503 \times 10^{27} h_{0}^{-1} \mathrm{~cm}$, where we take $h_{0}=1$, see Ref. [2]. 
Table 1. Values of $C$ and $\delta$ for some values of $D_{P}$. Time variation of space dimension today has also been calculated in terms of $\mathrm{yr}^{-1}$, see Ref. [2].

\begin{tabular}{cccc}
$D_{P}$ & $C$ & $\delta(\mathrm{cm})$ & $\left.\dot{D}_{t}\right|_{0}\left(\mathrm{yr}^{-1}\right)$ \\
\hline \hline 3 & $+\infty$ & 0 & 0 \\
\hline 4 & 1678.8 & $8.6 \times 10^{-216}$ & $-5.5 \times 10^{-13}$ \\
\hline 10 & 599.6 & $1.5 \times 10^{-59}$ & $-1.5 \times 10^{-12}$ \\
\hline 25 & 476.9 & $8.3 \times 10^{-42}$ & $-1.9 \times 10^{-12}$ \\
\hline$+\infty$ & 419.7 & $\ell_{P}$ & $-2.2 \times 10^{-12}$ \\
\hline \hline
\end{tabular}

\section{Running of the Spectral Index in Noncommutative Decrum- pling Inflation}

In this section, we present explicit and general formulae for the spectral index and its running within the framework of noncommutative decrumpling inflation. To do so, we first present the formalism of decrumpling chaotic inflation [4] in commutative spaces and then apply them in the context of noncommutative spaces. For the purposes of illustration, we apply our results for two classes of examples of the inflaton potential.

\subsection{Decrumpling chaotic inflation}

Inflation has been studied in the framework of decrumpling or TVSD model. The crucial equations are [4]

$$
\begin{aligned}
& H^{2}=\left(\frac{\dot{a}}{a}\right)^{2}=\frac{16 \pi}{D_{t}\left(D_{t}-1\right) M_{P}^{2}}\left(\frac{1}{2} \dot{\phi}^{2}+V(\phi)\right)-\frac{k}{a^{2}}, \text { Friedmann equation, } \\
& \ddot{\phi}+D_{t} H \dot{\phi}+\dot{D}_{t} \dot{\phi}\left(\ln \frac{a}{a_{0}}+\frac{d \ln V_{D_{t}}}{d D_{t}}\right)=-V^{\prime}(\phi), \quad \text { Fluid equation, }
\end{aligned}
$$

where $V_{D_{t}}$ is the volume of the space-like sections [2]

$$
V_{D_{t}}= \begin{cases}\frac{2 \pi^{\left(D_{t}+1\right) / 2}}{\Gamma\left[\left(D^{+}+1\right) / 2\right]}, & \text { if } k=+1, \quad \text { closed decrumpling model, } \\ \frac{\pi^{\left(D_{t} / 2\right)}}{\Gamma\left(D_{t} / 2+1\right)} \chi_{c}{ }^{D_{t}}, & \text { if } k=0, \quad \text { flat decrumpling model, } \\ \frac{2 \pi^{\left(D_{t} / 2\right)} f\left(\chi_{c}\right),}{\Gamma\left(D_{t} / 2\right)} f(\text { if } k=-1, \quad \text { open decrumpling model. }\end{cases}
$$

These volumes of space-like sections are valid even in the case of constant $D$-space [2]. Here $\chi_{C}$ is a cut-off and $f\left(\chi_{c}\right)$ is a function thereof [2].

Using the slow-roll approximation in decrumpling model [4]

$$
\begin{aligned}
\dot{\phi}^{2} & \ll V(\phi), \\
\ddot{\phi} & \ll D_{t} H \dot{\phi}, \\
-\dot{H} & \ll H^{2}
\end{aligned}
$$


and

$$
\dot{D}_{t}\left(\ln \frac{a}{a_{0}}+\frac{d \ln V_{D_{t}}}{d D_{t}}\right) \ll D_{t} H,
$$

Eqs. (3.1) and (3.2) can be rewritten for a flat decrumpling model, i.e. $k=0$, in the simpler set

$$
\begin{aligned}
H^{2} & \simeq \frac{16 \pi V(\phi)}{D_{t}\left(D_{t}-1\right) M_{P}^{2}}, \\
D_{t} H \dot{\phi} & \simeq-V^{\prime}(\phi) .
\end{aligned}
$$

Note that the slow-roll condition (3.7) has not been considered in Ref. [4]. The validity of this condition is obvious by regarding Eq. (2.5). Substituting (2.5) in (3.8), dynamics of the spatial dimension is given by [4]

$$
\dot{D}_{t}^{2} \simeq \frac{16 \pi D_{t}^{3} V(\phi)}{C^{2}\left(D_{t}-1\right) M_{P}^{2}}
$$

During inflation, $H$ is slowly varying in the sence that its change per Hubble time $\epsilon \equiv$ $-\dot{H} / H^{2}$ is less than one. The slow-roll condition $|\eta| \ll 1$ is actually a consequences of the condition $\epsilon \ll 1$ plus the slow-roll approximation $D_{t} H \dot{\phi} \simeq-V^{\prime}(\phi)$. Deferentiating (3.9) one finds

$$
\frac{\ddot{\phi}}{H \dot{\phi}}=\epsilon-\eta+\frac{D_{t}}{C},
$$

where the slow-roll parameters in decrumpling model are defined by

$$
\begin{gathered}
\epsilon \equiv \frac{\left(D_{t}-1\right) M_{P}^{2}}{32 \pi}\left(\frac{V^{\prime}}{V}\right)^{2}, \\
\eta \equiv \frac{\left(D_{t}-1\right) M_{P}^{2}}{16 \pi}\left(\frac{V^{\prime \prime}}{V}\right) .
\end{gathered}
$$

It should be emphasized that the slow-roll parameters in decrumpling model as presented in Ref. [4] are different from those given in (3.12) and (3.13). This difference is due to the slow-roll condition (3.7) which has not been considered in Ref. [4]. Furthermore, in the constant $D$-space, the slow-roll parameters (3.12) and (3.13) are also valid by substituting $D_{t}$ by $D$, see Ref. [4].

\subsection{Explicit formulae for running in noncommutative decrumpling inflation}

The density perturbation in noncommutative decrumpling inflation can be expressed by $[24]$

$$
\delta_{H}^{2}=\left(\frac{H}{2 \pi}\right)^{2}\left(1-\frac{3}{32} \frac{H^{4}}{\Lambda^{4}} \sin ^{2} \vartheta\right)^{2}\left(\frac{H}{\dot{\phi}}\right)^{2}
$$


where $\vartheta$ is the angle between the comoving wave-number $k$ and the third axis and $\Lambda^{-1}$ is the noncommutativity length scale [24, 25].

The density perturbation in noncommutative decrumpling inflation can be determined by substituting (3.8) and (3.9) in (3.14)

$$
\delta_{H}^{2}=\frac{9216 \pi V^{3}}{D_{t}^{3}\left(D_{t}-1\right)^{3} M_{P}^{6} V^{\prime 2}}\left(1-\frac{3}{32} \frac{H^{4}}{\Lambda^{4}} \sin ^{2} \vartheta\right)^{2} .
$$

This expression is evaluated at the horizon crossing time when $k=a H$. Since the value of Hubble constant does not change too much during inflationary epoch, we can obtain $d k=H d a$ and $d \ln k=H d t=d a / a$. Using the slow-roll condition in decrumpling inflation

$$
\frac{d}{d \ln k}=-\frac{V^{\prime}}{D_{t} H^{2}} \frac{d}{d \phi}
$$

and also the dimensional constraint of the model we have

$$
\begin{aligned}
\frac{d D_{t}}{d a} & =-\frac{D_{t}^{2}}{C a}, \\
\frac{d D_{t}}{d \ln k} & =-\frac{D_{t}^{2}}{C}, \\
\frac{d D_{t}}{d \phi} & =\frac{D_{t}^{3} H^{2}}{V^{\prime} C} .
\end{aligned}
$$

After a lengthy but straightforward calculation by using Eqs. (3.8), (3.9) and (3.15)(3.19) we find

$$
\begin{aligned}
n_{S}-1 & \equiv \frac{d \ln \delta_{H}^{2}}{d \ln k} \\
& =-6 \epsilon+2 \eta+\frac{6 \pi V^{\prime 2} \sin ^{2} \vartheta}{D_{t}^{2}\left(D_{t}-1\right) \Lambda^{4} M_{P}^{2}}\left(1-\frac{3}{32} \frac{H^{4}}{\Lambda^{4}} \sin ^{2} \vartheta\right)^{-1} \\
& \times\left[1-\frac{16 \pi D_{t}\left(2 D_{t}-1\right)}{C\left(D_{t}-1\right)^{2} M_{P}^{2}}\left(\frac{V}{V^{\prime}}\right)^{2}\right]+\frac{3 D_{t}\left(2 D_{t}-1\right)}{C\left(D_{t}-1\right)}
\end{aligned}
$$

where $\epsilon$ and $\eta$ are given in (3.12) and (3.13). To calculate the running we use the following expressions

$$
\begin{aligned}
\frac{d \epsilon}{d \ln k} & =-\frac{D_{t}^{2}}{C\left(D_{t}-1\right)} \epsilon-2 \epsilon \eta+4 \epsilon^{2}, \\
\frac{d \eta}{d \ln k} & =-\frac{D_{t}^{2}}{C\left(D_{t}-1\right)} \eta+2 \epsilon \eta-\xi
\end{aligned}
$$

where the third slow-roll parameter is defined by

$$
\xi \equiv \frac{\left(D_{t}-1\right)^{2} M_{P}^{4}}{(16 \pi)^{2}}\left(\frac{V^{\prime} V^{\prime \prime \prime}}{V^{2}}\right)
$$


Using above equations, running in noncommutative decrumpling inflation has this explicit expression

$$
\begin{aligned}
& \frac{d n_{S}}{d \ln k}=16 \epsilon \eta-24 \epsilon^{2}-2 \xi+\frac{6 D_{t}^{2}}{C\left(D_{t}-1\right)} \epsilon-\frac{2 D_{t}^{2}}{C\left(D_{t}-1\right)} \eta-\frac{3 D_{t}^{2}\left(2 D_{t}^{2}-4 D_{t}+1\right)}{C^{2}\left(D_{t}-1\right)^{2}} \\
& -\frac{6 \sin ^{2} \vartheta}{D_{t}^{2}\left(D_{t}-1\right) \Lambda^{4}}\left(1-\frac{3}{32} \frac{H^{4}}{\Lambda^{4}} \sin ^{2} \vartheta\right)^{-2}\left[\frac { 3 \pi ^ { 2 } V ^ { \prime 2 } \operatorname { s i n } ^ { 2 } \vartheta } { M _ { P } ^ { 4 } \Lambda ^ { 4 } } \left(\frac{V^{\prime 2}}{D_{t}^{2}\left(D_{t}-1\right)}\right.\right. \\
& -\frac{32 \pi\left(2 D_{t}-1\right) V^{2}}{C D_{t}\left(D_{t}-1\right)^{3} M_{P}^{2}}+\frac{256 \pi^{2}\left(2 D_{t}-1\right)^{2} V^{4}}{C^{2}\left(D_{t}-1\right)^{5} M_{P}^{4} V^{\prime 2}}+\left(1-\frac{3}{32} \frac{H^{4}}{\Lambda^{4}} \sin ^{2} \vartheta\right) \\
& \left.\times\left(\frac{V^{\prime 2} V^{\prime \prime}\left(D_{t}-1\right)}{8 V}-\frac{\pi V^{\prime 2} D_{t}\left(7 D_{t}-4\right)}{C\left(D_{t}-1\right) M_{P}^{2}}-\frac{16 \pi^{2} V^{2} D_{t}^{2}\left(6 D_{t}^{2}-2 D_{t}-1\right)}{C^{2}\left(D_{t}-1\right)^{3} M_{P}^{4}}\right)\right] .
\end{aligned}
$$

Let us now use (3.20) and (3.24) for two potentials of the inflation. We first study $V(\phi)=m^{2} \phi^{2} / 2$ and then $V(\phi)=\lambda \phi^{4}$.

3.3 The first example: $V(\phi)=\frac{1}{2} m^{2} \phi^{2}$

For the purposes of illustration, we now consider the potential $V(\phi)=m^{2} \phi^{2} / 2$

$$
\begin{aligned}
& \epsilon=\eta=\frac{\left(D_{t}-1\right) M_{P}^{2}}{8 \pi \phi^{2}}, \\
& \xi=0 .
\end{aligned}
$$

Using the definition of e-folding in decrumpling inflation [4]

$$
\mathcal{N}=-\frac{16 \pi}{M_{P}^{2}} \int_{\phi}^{\phi_{f}} \frac{V}{\left(D_{t}-1\right) V^{\prime}} d \phi
$$

we have

$$
\mathcal{N}=\frac{4 \pi}{\left(D_{t}-1\right) M_{P}^{2}}\left(\phi^{2}-\phi_{f}^{2}\right)
$$

where $\phi_{f}$ is the value of the inflaton field at the end of inflation. Note that in the integral of (3.27), $D_{t}$ is as a function of the inflaton field. To integrate in (3.27) we take $D_{t}$ to be independent on the inflaton field because the relationship between $D_{t}$ and $\phi$ in decrumpling inflation is too weak [5]. For this reason, our approximation is appropriate for integration of Eq. (3.27).

To obtain e-folding as a function of the inflaton field, we must obtain $\phi_{f}$. From $\epsilon=\eta=1$ we get

$$
\phi_{f}=\sqrt{\frac{D_{t}-1}{8 \pi}} M_{P} .
$$


From (3.28) and (3.29), we have

$$
\phi^{2}=\frac{\left(D_{t}-1\right) M_{P}^{2}}{8 \pi}(2 \mathcal{N}+1)
$$

With no loss of generality, we take $\vartheta=\frac{\pi}{2}$. So substituting $\sin \vartheta=1$ in the above equations leads us to

$$
\begin{aligned}
n_{S}-1 & =-\frac{4}{(2 \mathcal{N}+1)}+\frac{3(2 \mathcal{N}+1) m^{4}}{4 D_{t}^{2} \Lambda^{4}}\left(1-\frac{3(2 \mathcal{N}+1)^{2} m^{4}}{32 D_{t}^{2} \Lambda^{4}}\right)^{-1} \\
& \times\left[1-\frac{D_{t}\left(2 D_{t}-1\right)(2 \mathcal{N}+1)}{2 C\left(D_{t}-1\right)}\right]+\frac{3 D_{t}\left(2 D_{t}-1\right)}{C\left(D_{t}-1\right)} \\
\frac{d n_{S}}{d \ln k} & =-\frac{8}{(2 \mathcal{N}+1)^{2}}-\frac{4 D_{t}^{2}}{C\left(D_{t}-1\right)(2 \mathcal{N}+1)}-\frac{3 D_{t}^{2}\left(2 D_{t}^{2}-4 D_{t}+1\right)}{C^{2}\left(D_{t}-1\right)^{2}} \\
& -\frac{3 m^{4}}{2 D_{t}^{2}\left(D_{t}-1\right) \Lambda^{4}}\left(1-\frac{3(2 \mathcal{N}+1)^{2} m^{4}}{32 D_{t}^{2} \Lambda^{4}}\right)^{-2}\left[\frac{3 m^{4}\left(D_{t}-1\right)^{2}(2 \mathcal{N}+1)^{2}}{16 \Lambda^{4}}\right. \\
& \times\left(\frac{1}{D_{t}^{2}\left(D_{t}-1\right)}-\frac{\left(2 D_{t}-1\right)(2 \mathcal{N}+1)}{C D_{t}\left(D_{t}-1\right)^{2}}+\frac{\left(2 D_{t}-1\right)^{2}(2 \mathcal{N}+1)^{2}}{4 C^{2}\left(D_{t}-1\right)^{3}}\right) \\
& +\left(1-\frac{3(2 \mathcal{N}+1)^{2} m^{4}}{32 D_{t}^{2} \Lambda^{4}}\right)\left(D_{t}-1-\frac{D_{t}\left(7 D_{t}-4\right)(2 \mathcal{N}+1)}{2 C}\right. \\
& \left.-\frac{D_{t}^{2}\left(6 D_{t}^{2}-2 D_{t}-1\right)(2 \mathcal{N}+1)^{2}}{4 C^{2}\left(D_{t}-1\right)}\right)
\end{aligned}
$$

The point is that the general formulae (3.31) and (3.32) in terms of unknown value of $\vartheta$ could be obtained by substituting $\Lambda^{2}$ with $\Lambda^{2} / \sin \vartheta$.

\subsection{The second example: $V(\phi)=\lambda \phi^{4}$}

For the second example, we study $V(\phi)=\lambda \phi^{4}$. The slow-roll parameters are

$$
\begin{aligned}
\epsilon & =\frac{\left(D_{t}-1\right) M_{P}^{2}}{2 \pi \phi^{2}}, \\
\eta & =\frac{3\left(D_{t}-1\right) M_{P}^{2}}{4 \pi \phi^{2}}, \\
\xi & =\frac{3\left(D_{t}-1\right)^{2} M_{P}^{4}}{8 \pi^{2} \phi^{4}} .
\end{aligned}
$$

Using the definition of e-folding in inflation, we have

$$
\mathcal{N}=\frac{2 \pi}{M_{P}^{2}\left(D_{t}-1\right)}\left(\phi^{2}-\phi_{f}^{2}\right)
$$


To obtain the e-folding number as a function of the inflaton field, we must obtain the value of the inflaton field at the end of inflation. From $\epsilon=1$ we get

$$
\phi_{f}=\sqrt{\frac{D_{t}-1}{2 \pi}} M_{P} .
$$

Assuming $\eta=1$ and $\xi=1$, we obtain

$$
\phi_{f}=\sqrt{\frac{3\left(D_{t}-1\right)}{4 \pi}} M_{P}
$$

and

$$
\phi_{f}=\left(\frac{3\left(D_{t}-1\right)^{2}}{8 \pi^{2}}\right)^{1 / 4} M_{P},
$$

respectively. These values of $\phi_{f}$ based on the condition $\eta=1$ and $\xi=1$ are larger than $\phi_{f}$ arisen from $\epsilon=1$. We here take the condition $\epsilon=1$ by itself is a true condition to obtain $\phi_{f}$. From (3.36) and (3.37) we have

$$
\phi^{2}=\frac{\left(D_{t}-1\right)(\mathcal{N}+1) M_{P}^{2}}{2 \pi} .
$$

Again we take $\vartheta=\pi / 2$. Substituting $\sin \vartheta=1$ in (3.20) and (3.24) and using (3.33), (3.34) and (3.35) we obtain the main formulae for the spectral index and its running

$$
\begin{aligned}
& n_{S}-1=-\frac{3}{(\mathcal{N}+1)}+\frac{12 \lambda^{2} M_{P}^{4}\left(D_{t}-1\right)^{2}(\mathcal{N}+1)^{3}}{\pi^{2} D_{t}^{2} \Lambda^{4}} \\
& \times\left(1-\frac{3 \lambda^{2} M_{P}^{4}\left(D_{t}-1\right)^{2}(\mathcal{N}+1)^{4}}{2 \pi^{4} D_{t}^{2} \Lambda^{4}}\right)^{-1}\left[1-\frac{D_{t}\left(2 D_{t}-1\right)(\mathcal{N}+1)}{2 C\left(D_{t}-1\right)}\right] \\
& +\frac{3 D_{t}\left(2 D_{t}-1\right)}{C\left(D_{t}-1\right)}, \\
& \frac{d n_{S}}{d \ln k}=-\frac{3}{(\mathcal{N}+1)^{2}}+\frac{3 D_{t}^{2}}{C\left(D_{t}-1\right)(\mathcal{N}+1)}-\frac{24 \lambda^{2} M_{P}^{4}\left(D_{t}-1\right)(\mathcal{N}+1)^{2}}{\pi^{2} D_{t}^{2} \Lambda^{4}} \\
& \times\left(1-\frac{3 \lambda^{2} M_{P}^{4}\left(D_{t}-1\right)^{2}(\mathcal{N}+1)^{4}}{2 \pi^{2} D_{t}^{2} \Lambda^{4}}\right)^{-2}\left\{\frac{3 \lambda^{2} M_{P}^{4}\left(D_{t}-1\right)^{4}(\mathcal{N}+1)^{4}}{\pi^{2} \Lambda^{4}}\right. \\
& \times\left[\frac{1}{D_{t}^{2}\left(D_{t}-1\right)}-\frac{\left(2 D_{t}-1\right)(\mathcal{N}+1)}{C D_{t}\left(D_{t}-1\right)^{2}}+\frac{\left(2 D_{t}-1\right)^{2}(\mathcal{N}+1)^{2}}{4 C^{2}\left(D_{t}-1\right)^{3}}\right] \\
& +\left(1-\frac{3 \lambda^{2} M_{P}^{4}\left(D_{t}-1\right)^{2}(\mathcal{N}+1)^{4}}{2 \pi^{2} \Lambda^{4} D_{t}^{2}}\right)\left[\frac{3\left(D_{t}-1\right)}{2}-\frac{D_{t}\left(7 D_{t}-4\right)(\mathcal{N}+1)}{2 C}\right. \\
& -\frac{D_{t}^{2}\left(6 D_{t}^{2}-2 D_{t}-1\right)(\mathcal{N}+1)^{2}}{4 C^{2}\left(D_{t}-1\right)}-\frac{3 D_{t}^{2}\left(2 D_{t}^{2}-4 D_{t}+1\right)}{C^{2}\left(D_{t}-1\right)^{2}} .
\end{aligned}
$$




\section{Conclusions}

We have studied the effects of both space-space noncommutative geometry and time variability of spatial dimensions on the spectral index and its running, and obtained the correction terms to the standard formulae. If there exists any time variability of spatial dimensions and noncommutativity of space in nature, as it seems to emerge from different theories and arguments, its implications should appear in the spectral index and its running. We presented the general features of our formalism and applied it to two specific potentials of chaotic inflation. The results show that the terms arising from noncommutativity of space depend on the space-space noncommutativity length scale and those arising from time variability of spatial dimensions depend on the universal parameter of decrumpling model.

For the purposes of illustration, we present the formulae for the spectral index and its running in noncommutative decrumpling inflation for two classes of examples of the inflaton potentials. The first one is $m^{2} \phi^{2} / 2$ and the second $\lambda \phi^{4}$.

Since the upper value of the universal parameter, $C$, of decrumpling model is about 1680, the correction terms arising from time variability of spatial dimensions, being of the order of $\mathcal{O}(1 / C)$ or its powers of 2 or so, are of the order of $10^{-3}$ or less. On the other hand, considering the noncommutativity length scale $\Lambda^{-1}$, being of the order of the Planck length, so we obtain $M_{P}^{4} / \Lambda^{4}$ of the order of unity, $\mathcal{O}(1)$. This point simplifies the formulae of the spectral index and its running, see Eqs. (3.41) and (3.42). We know the mass of the inflaton field to be $m \simeq 1.21 \times 10^{-6} M_{P}$, the coupling constant $\lambda \simeq 10^{-15}$ and the e-folding number $\mathcal{N} \simeq 60$. Regarding these values of $m, \lambda$ and $\mathcal{N}$, and comparing the correction terms due to time variability of spatial dimensions with those due to space-space noncommutative geometry, we conclude that the effects of noncommutative spaces on the spectral index and its running are much smaller than the effects of time variability of spatial dimensions. When $C \rightarrow+\infty$ and $D_{t}=D_{0}=3$ our results in this article reproduce the standard formulae in noncommutative threespace dimensions, as presented in Ref. [24].

Considering the accuracy of the WMAP data which is up to two or three decimal integers $[26,27]$, we conclude that the effects of space-space noncommutative geometry on the spectral index and its running cannot be currently detected while the effects of time variability of spatial dimensions can be detected by the present experimental celectial or ground instruments.

The final point that must be emphasized is about decrumpling model in cosmology. The original motivation of this model presented in the pioneer paper [1] was based on an ad hoc assumption inspired from polymer physics. It is quite possible that this part of decrumpling model should be revised. However, just how this should be done is 
far from obvious. The progress in decrumpling model can only be made if there is a

breakthrough in terms of finding a natural mechanism for varying the spatial dimension in some alternative fashion to that which we have considered.

\section{Acknowledgments}

It is a pleasure to thank Malcolm MacCallum, Michael Murphy and Jean-Philippe Uzan for helpful comments. F.N. thanks Amir and Shahrokh for useful helps.

\section{References}

[1] M. Khorrami, R. Mansouri and M. Mohazzab, Helv. Phys. Acta 69, 237 (1996), [gr-qc/9607049]; M. Khorrami, R. Mansouri, M. Mohazzab and M. R. Ejtehadi, "A model Universe with variable dimension: Expansion as decrumpling", [gr-qc/9507059].

[2] R. Mansouri and F. Nasseri, Phys. Rev. D60, 123512 (1999), [gr-qc/9902043], and references therein.

[3] R. Mansouri, F. Nasseri and M. Khorrami, Phys. Lett. A259, 194 (1999), [gr-qc/9905052].

[4] F. Nasseri and S. Rahvar, Int. J. Mod. Phys. D11, 511 (2002), [gr-qc/0008044].

[5] F. Nasseri, Phys. Lett. B538, 223 (2002), [gr-qc/0203032].

[6] J.A.S. Lima and M. Mohazzab, Int. J. Mod. Phys. D7, 657 (1998), [gr-qc/9607017].

[7] M. Mohazzab and J.A.S. Lima, Int. J. Mod. Phys. D8, 751 (1999).

[8] J.M. Cline and J. Vinet, Phys. Rev. D68, 025015 (2003), [hep-ph/0211284].

[9] J.D. Barrow, Phys. Rev. D35, 1805 (1987).

[10] N. Seiberg and E. Witten, JHEP 9909, 032 (1999), [hep-th/9908142].

[11] A. Sitarz, JHEP 0209, 034 (2002), [math-ph/0206040].

[12] M. Chaichian, N. Nishijima and A. Tureanu, Phys. Lett. B568, 146 (2003), [hep-th/0209008].

[13] R.J. Szabo, Phys. Rept. 378, 207 (2003), [hep-th/0109162].

[14] S.A. Alavi, Phys. Scripta 67, 366 (2003), [hep-th/0208098]. 
[15] S.A. Alavi, Phys. Scripta (in press), "Heisenberg quantization for systems of identical particles and the Pauli exclusion principle in noncommutative spaces", [hep-th/0208007].

[16] C. Zachos, Mod. Phys. Lett. A19, 1483 (2004), [hep-th/0402127].

[17] D. Kamani, Mod. Phys. Lett. A18, 2125 (2004), [hep-th/0308017].

[18] A. Torrielli, Mod. Phys. Lett. A18, 2525 (2003), [hep-th/0307089].

[19] R. Szabo, Int. J. Mod. Phys. A19, 1837 (2004), [physics/0401142].

[20] Q.G. Huang and M. Li, JCAP 0311, 001 (2003), [astro-ph/0308458].

[21] S. Tsujikawa, R. Maartens and R. Brandenberger, Phys. Lett. B574, 141 (2003).

[22] R.G. Cai, Phys. Lett. B593, 1 (2004), [hep-th/0403134].

[23] M. Fukuma, Y. Kono and A. Miwa, Nucl. Phys. B682, 377 (2004), [hep-th/0307029].

[24] S.A. Alavi and F. Nasseri, "Running of the spectral index in noncommutative inflation", [astro-ph/0406477], accepted for publication in Int. J. Mod. Phys. A.

[25] F. Lizzi, G. Mangano, G. Miele and M. Peloso, JHEP 0206, 049 (2002), [hep-th/0203099].

[26] D.N. Spergel et al, Astrophys. J. Suppl. 148, 175 (2003), [astro-ph/0302209].

[27] H.V. Peiris et al, Astrophys. J. Suppl. 148, 213 (2003), [astro-ph/0302225]. 